\title{
Extinción y renovación de la memoria espacial en humanos*
}

\section{Extinction and renewal of spatial memory in humans}

DOI: 10.11144/Javeriana.upsy15-5.erme

Recepción: 24 Abril 2016 | Aprobación: 01 Noviembre 2016

\author{
DAVID LUNA ${ }^{\mathrm{a}}$ \\ Instituto Politécnico Nacional, México \\ Moisés Manzanares-Silva \\ Instituto Politécnico Nacional, México \\ Katia Rodríguez-GonzÁlez \\ Universidad Nacional Autónoma de México, México \\ Rodrigo Carranza-Jasso \\ Universidad Autónoma de Aguascalientes, México
}

\begin{tabular}{l}
\hline $\begin{array}{l}\text { aAutor de correspondencia. } \\
\text { dglunap@ipn.mx }\end{array}$ \\
Para citar este artículo: Luna, D., Rodriguez- \\
González, K., \& Carranza-Jasso, R. (2016). \\
Extinción y renovación de la memoria espacial en \\
humanos. Universitas Psychologica, 15(5). http:// \\
dx.doi.org/10.11144/Javeriana.upsy15-5.erme
\end{tabular}

dx.doi.org/10.11144/Javeriana.upsy15-5.erme

\section{RESUMEN}

Participantes humanos fueron entrenados en un laberinto virtual de agua para localizar en dos fases sucesivas una plataforma cuya ubicación fue específica a la fase vigente. Posteriormente realizaron una prueba sin plataforma. El Grupo ABA realizó la primera fase y la prueba en un contexto A, y la segunda fase en un contexto B. El Grupo ABB realizó la primera fase en el contexto A, y la segunda fase y la prueba en el contexto B. Ambos grupos localizaron la plataforma en cada fase. Durante la prueba, la última ubicación de la plataforma fue preferida por el Grupo $\mathrm{ABB}$, mientras que la primera ubicación fue preferida por el Grupo ABA. Estos resultados indican renovación de la memoria espacial en humanos.

\section{Palabras clave}

aprendizaje asociativo; laberinto virtual de agua; preferencia espacial; extinción; efectos de recuperación.

\begin{abstract}
Human participants were trained in a virtual water maze throughout two immediate phases, in order to locate a platform which particular location was specific to each phase. Afterwards, a trial without the platform was conducted.The ABA group experienced phase 1 and the test trial in Context A, while the second phase occurred in Context B. The ABB group received the first phase in Context $A$ and the phase 2 and test trial training in Context B. Both groups had to locate the platform in each phase. During the test trial, the last platform location was preferred by the $A B B$ group but the first platform location was preferred by the group ABA. These results suggest renewal of spatial memory in humans.

Keywords

associative learning; virtual water maze; spatial preference; extinction; recovery effects.
\end{abstract}

\section{Introducción}

Adquisición y extinción son procesos de aprendizaje que facilitan la adaptación de los organismos a su entorno 
(Méndez-Couz, Conejo, Vallejo \& Arias, 2015). En el primero de ellos, información novedosa, y su conducta asociada, es adquirida, retenida y posteriormente recuperada de la memoria (Hardt, Wang \& Nader, 2009); en el segundo, información antes relevante es ahora suprimida debido a su escaso valor adaptativo (Quirk \& Mueller, 2008). Ambos procesos han sido extensamente demostrados en animales humanos (Kinloch, Foster \& McEwan, 2009) y no humanos (Rescorla, 2001), y una de las aproximaciones teóricas más fructíferas en su estudio ha sido la del aprendizaje asociativo (Mackintosh, 1983).

En las últimas décadas, el aprendizaje asociativo ha sido empleado también para explicar los mecanismos cognitivos que subyacen a la adquisición de la memoria espacial en especies diversas, incluyendo la humana (Leising \& Blaisdell, 2009; pero ver Hardt, Hupbach \& Nadel, 2009). La memoria espacial permite a los organismos desplazarse funcionalmente en su entorno (Hampson, 1995), y aunque su adquisición es de alta relevancia evolutiva (Pravosudov \& Roth II, 2013), su extinción es también vital para la adaptación al medio (Devenport, 1984). A pesar de lo anterior, los mecanismos de aprendizaje que operan en la extinción de la memoria espacial han sido poco investigados y en consecuencia, el desarrollo teórico de este efecto es actualmente escaso (Lattal, Mullen \& Abel, 2003).

Desde la aproximación del aprendizaje asociativo, la extinción puede ser interpretada como producto de la pérdida de la información almacenada en memoria (Rescorla \& Wagner, 1972). No obstante, puede ser también considerada como consecuencia de la adquisición de una nueva memoria, de naturaleza inhibitoria, que interfiere con la recuperación de una memoria previamente adquirida (Bouton, 1993). A favor de esta última postura, existe evidencia de la recuperación de una respuesta extinguida cuando una prueba se realiza tras un intervalo de retención (i.e., recuperación espontánea; Pavlov, 1927) o en un contexto diferente al presente durante la extinción (i.e., renovación; Bouton \& King, 1983). Diversas demostraciones de recuperación espontánea de la memoria espacial con animales humanos (Luna \& Martínez, 2015) y no humanos (Lattal et al., 2003; Leising, Wong \& Blaisdell, 2015; Prados, Manteiga \& Sansa, 2003) sugieren la participación del aprendizaje asociativo en la extinción de la memoria espacial. Sin embargo, existe también evidencia de la ausencia de este efecto (Méndez-Couz, Conejo, Vallejo \& Arias, 2014; Rossato, Bevilaqua, Medina, Izquierdo \& Cammarota, 2006), lo cual es consistente con posturas no asociativas de la adquisición y extinción de la memoria espacial (Burgess, Jackson, Hartley \& O'Keefe, 2000; Foster, Morris \& Dayan, 2000; O'Keefe \& Nadel, 1978). No es del todo claro si dicha controversia obedece al proceso de aprendizaje mismo (i.e., asociativo vs. no asociativo) o debido a aspectos metodológicos propios a los estudios conducidos (para una discusión ver Luna \& Martínez, 2015). Sin embargo, la participación del aprendizaje asociativo en la extinción de la memoria espacial se ve favorecida por evidencia que demuestra su renovación en roedores (Lattal et al., 2003; Prados et al., 2003), aunque actualmente no hay datos disponibles sobre la ocurrencia de este mismo efecto en humanos u otras especies.

El experimento aquí reportado fue diseñado para evaluar la ocurrencia de un efecto de renovación de la memoria espacial en humanos entrenados en un laberinto virtual de agua (LVA; Hardt et al., 2009). Tras la adquisición de una memoria espacial original acerca de la ubicación de una meta, los participantes fueron expuestos a un procedimiento de extinción por reversión en el cual dicha meta fue reubicada en un sitio novedoso (Lattal et al., 2003). Posteriormente se realizó una prueba en el mismo contexto en que se condujo la extinción por reversión o en uno diferente. Si mecanismos propios al aprendizaje asociativo participan en la extinción de la memoria espacial humana, entonces una prueba realizada un contexto diferente al presente durante la extinción debería ocasionar la renovación de la memoria espacial originalmente adquirida. La demostración de este efecto indicaría una continuidad cognitiva (Wasserman, 1993) entre animales humanos y 
no humanos en la adquisición y extinción de la memoria espacial.

\section{Método}

\section{Participantes}

Colaboraron 32 estudiantes de pregrado (edad promedio $=20.37$ años, D.E. $=2.49 ; 50 \%$ 우), divididos en dos grupos $(\mathrm{n}=16)$ igualados por sexo (i.e., Grupo ABA y Grupo ABB). Los participantes fueron ingenuos en los objetivos del experimento, contaban con un buen estado de salud general y con vista normal o corregida, y no consumían algún fármaco o sustancia que afectara su desempeño cognitivo o motor. Previa la realización del estudio todos los participantes fueron informados de sus derechos de acuerdo a la Declaración de Helsinski (W. M. A., 2008).

\section{Ambiente experimental}

El experimento se realizó en un salón de 2 x $3 \mathrm{~m}$ amueblado con una silla y un escritorio sobre el cual se colocó una computadora IBM compatible con la que se ejecutó la tarea experimental. Una vez sentados, la vista de los participantes estuvo a $60 \mathrm{~cm}$ aproximadamente del monitor de la computadora.

\section{Tarea experimental}

La tarea experimental consistió en un LVA diseñado con el programa Maze Suite v. 2.3.0.1 (Ayaz et al., 2011), el cual presentó en perspectiva de primera persona una alberca octagonal rodeada por un muro sobre el cual se colocó en forma distribuida un conjunto de claves discretas que fueron figuras geométricas tridimensionales (E: esfera; C: cubo; PR: paralelepípedo rectangular) de diferentes colores (Am: amarillo; Az: azul; V: verde; R: rojo). La alberca se ubicó en una habitación con muros de colores y texturas diferentes, y con un cielo nublado a manera de plafón. Los elementos de la habitación y el conjunto de claves discretas fungieron como claves distales útiles para localizar una plataforma cuadrada oculta debajo del nivel del agua y cuyo tamaño fue de aproximadamente el 3.5\% del área total de la alberca. Se diseñaron dos versiones del LVA a fin de ser utilizadas como contextos virtuales. En una de ellas el muro de la alberca fue gris y el color del agua fue azul claro (contexto virtual A), y en la otra el muro fue rosa con una franja amarilla y el agua fue azul oscuro (contexto virtual B). Los elementos restantes fueron comunes a ambos contextos (ver Figura 1). El movimiento en el LVA fue controlado con las flechas de dirección en el teclado de la computadora: $(\uparrow)$ nado adelante y $(\downarrow)$ nado atrás, para la rotación sobre el eje del participante se utilizó $(\leftarrow)$ vuelta a la izquierda y $\rightarrow$ ) vuelta a la derecha (Hardt et al., 2009). Un giro de $360^{\circ}$ tomó aproximadamente $5 \mathrm{s.}$ mientras que cruzar la alberca de un muro a otro requirió alrededor de $7 \mathrm{~s}$.

\section{Figura 1}

Laberinto virtual de agua

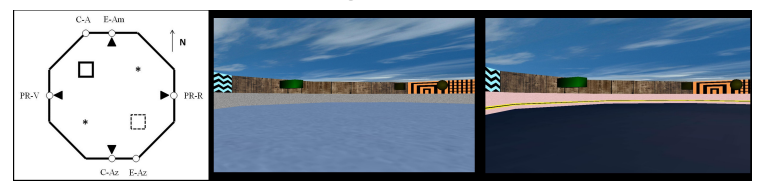

Izquierda: Vista esquemática del laberinto virtual de agua diseñado. El recuadro interno sólido y punteado indica la ubicación de la plataforma durante la Fase de Discriminación y la Fase de Reversión, respectivamente. Las flechas al centro de los muros son los puntos de partida empleados durante el entrenamiento y los asteriscos corresponden a los empleados para la prueba. Las letras en la leyenda junto a cada círculo abierto señalan la figura geométrica tridimensional empleada (E: esfera; C: cubo; PR: paralelepípedo rectangular) y su color (A: amarillo; Az: azul; V: verde; R: rojo). Centro y derecha respectivamente: Vista del laberinto virtual de agua desde la perspectiva del participante en el contexto A y contexto B. Fuente: elaboración propia.

\section{Procedimiento}

El experimento consistió de una sesión de 15 min aproximadamente, realizada en forma individual. Un investigador condujo a cada participante al ambiente experimental y los acompañó durante 
una Fase de Práctica y una de Pre-entrenamiento a fin de entregarles las instrucciones impresas en una hoja de papel. La Fase de Práctica incluyó un ensayo único de $30 \mathrm{~s}$ de duración, para el cual se solicitó a los participantes desplazarse libremente en el LVA utilizando las flechas del teclado de la computadora. La Fase de Pre-entrenamiento comprendió dos ensayos en los que se solicitó a los participantes hacer contacto con una plataforma visible sobre el nivel del agua ubicada en un punto específico del LVA. En ambas fases se empleó el entorno virtual utilizado por Luna y Martínez (2015) en las fases homónimas de su estudio. Una vez finalizadas dichas fases, se entregó a los participantes las instrucciones propias al entrenamiento, las cuales indicaban que debían localizar una plataforma oculta en dos albercas diferentes, cada una ubicada en un balneario (contexto virtual) distinto. Tras aclarar cualquier duda el investigador se retiró del ambiente experimental. El entrenamiento comprendió una Fase de Discriminación y una Fase de Reversión, cada una de 8 ensayos con una duración máxima de 60 s y realizada en un contexto virtual específico (A o B). Para cada ensayo, si la plataforma era localizada se presentó un mensaje al centro de la pantalla de la computadora con la leyenda "Aquí está la plataforma", en caso contrario el mensaje "Has muerto" era mostrado. Por presionar la tecla Enter en el teclado de la computadora los participantes podían programar el ensayo o fase siguiente según fuera el caso. La plataforma se localizó en el cuadrante Noroeste durante la Fase de Discriminación, y fue movida al cuadrante Sureste para la Fase de Reversión. Se emplearon cuatro puntos de partida programados semi-aleatoriamente con la restricción de no utilizar el mismo punto en forma consecutiva. Los contextos fueron contrabalanceados de forma que para la mitad de los participantes de cada sexo en cada grupo el contexto virtual A mostrara el agua azul claro con los muros grises y el contexto virtual B mostrara el agua azul oscuro con los muros rosa con franja amarilla. Para los participantes restantes la situación fue inversa. Al término del entrenamiento se realizó una Fase de Prueba, que consistió en un ensayo de
$60 \mathrm{~s}$ sin plataforma cuyo punto de partida fue al centro del cuadrante Suroeste para la mitad de los participantes de cada sexo por grupo, y el cuadrante Noreste para los participantes restantes (ver Figura 1). Antes del primer ensayo de cada fase, incluida la Fase de Prueba con su ensayo único, apareció por $10 \mathrm{~s}$ al centro de la pantalla de la computadora una leyenda con el nombre del balneario (contexto virtual) en el que los participantes se localizaban. El Grupo $\mathrm{ABB}$ realizó la Fase de Discriminación en un contexto diferente respecto al empleado para las fases restantes (i.e., Fase de Reversión y Fase de Prueba), mientras que el Grupo ABA realizó la Fase de Reversión en un contexto distinto respecto al empleado para las otras dos.

\section{Análisis de resultados}

Se registró para cada fase del entrenamiento y para la Fase de Prueba respectivamente, la latencia para localizar la plataforma y la permanencia en los cuadrantes reforzados. El análisis estadístico de los datos se condujo con el programa SPSS v.20 e incluyó pruebas t y análisis de varianza (ANOVA), con la prueba post hoc de la Diferencia Honestamente Significativa de Tuckey (DHS). Se calculó la d de Cohen (pruebas t) y la eta cuadrada parcial $\left(\# \mathrm{p}^{2}\right.$; ANOVA) como índices del tamaño del efecto, considerando un efecto pequeño, mediano y grande una $\mathrm{d} \geq \mathrm{a}$ $0.20,0.50$, y 0.80 , y una $\#^{2}$ p $\geq$ a $0.01,0.06$, 0.14 respectivamente (Aron \& Aron, 2001). Un resultado se consideró significativo a un valor de $\mathrm{p}<.05$.

\section{Resultados}

Los participantes disminuyeron su latencia para localizar la plataforma en cada fase del entrenamiento (Figura 2). Esto fue confirmado por conducir dos ANOVA $2 \times 2 \times(8)$, uno por cada fase, con los factores grupo (ABA vs. $\mathrm{ABB})$, sexo (hombres vs. mujeres) y ensayos $(1-8)$, los cuales revelaron un efecto principal para el factor ensayos [Fase de Discriminación: 
$\mathrm{F}(7,196)=13.23, \mathrm{p}<0.01, \#^{2} \mathrm{p}=0.32 ;$ Fase de Reversión: F $(7,196)=11.68, \mathrm{p}<$ 0.01, $\left.\#^{2} \mathrm{p}=0.29\right]$. Comparaciones múltiples post hoc (DHS) mostraron diferencias entre el Ensayo 1 y el Ensayo 8 de cada fase $(\mathrm{p}<0.01)$. Adicionalmente, para la Fase de Reversión se detectó una interacción Grupo x Ensayos, F (7, 196) $=2.5, p=0.01, \#^{2} p=0.08$, cuyo análisis de efectos simples confirmó una diferencia entre grupos solo en el ensayo 3, F $(1,24)=8.36$, p $<0.01, \#^{2} \mathrm{p}=.25$. El conjunto de estos datos indica que los participantes de ambos grupos aprendieron en cada fase la ubicación de la plataforma oculta, llegando a la Fase de Prueba en igualdad de condiciones.

\section{Figura 2}

Latencia para localizar la plataforma.

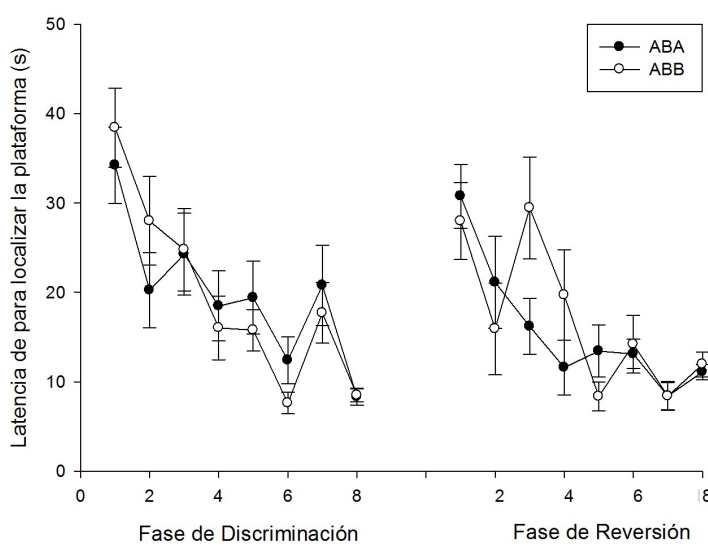

Latencia promedio para localizar la plataforma en cada fase del entrenamiento para el Grupo ABB (círculo vacío), y el Grupo ABA (círculo sólido). La barra de error indica el error estándar de la media. Fuente: elaboración propia.

Durante la Fase de Prueba, pruebas t de una cola para un grupo revelaron una permanencia por encima del nivel del azar (15 s) en el cuadrante reforzado en la Fase de Reversión para el Grupo ABB, t $(15)=3.60, \mathrm{p}<0.01$, $\mathrm{d}=1.85$, y en el reforzado durante la Fase de Discriminación para el Grupo ABA, t(15) $=2.53, \mathrm{p}=0.01, \mathrm{~d}=1.30$. Adicionalmente, este último grupo mostró una permanencia por debajo de lo esperado por azar en el cuadrante reforzado durante la Fase de Reversión, t(15)
$=3.58, \mathrm{p}<0.01, \mathrm{~d}=1.84$. Un ANOVA 2 grupos x 2 cuadrantes reforzados reveló una interacción Grupo x Cuadrante reforzado, $\mathrm{F}(1,60)=28.60, \mathrm{p}<0.01, \#^{2} \mathrm{p}=0.32$. Esta interacción fue analizada por conducir dos ANOVA unifactoriales. El primero comparó intra-grupo la permanencia en cada cuadrante en que se ubicó la plataforma, y reveló que el Grupo ABA permaneció más tiempo en el cuadrante reforzado durante la Fase de Discriminación, $\mathrm{F}(1,30)=16.01, \mathrm{p}<0.01, \# 2 \mathrm{p}=.34 ;$ mientras que el Grupo ABB permaneció menos tiempo en dicho cuadrante, $\mathrm{F}(1,30)=12.81$, $\mathrm{p}<0.01, \# 2 \mathrm{p}=0.29$. El segundo análisis reveló diferencias entre grupos en la permanencia en el cuadrante reforzado durante la Fase de Discriminación, $\mathrm{F}(1,30)=7.66, \mathrm{p}=0.01, \#^{2} \mathrm{p}$ $=0.20$; y durante la Fase de Reversión, F(1, $30)=24.49, p<0.01, \#^{2} p=0.44$. Estos datos indican una preferencia diferenciada de cada grupo por los cuadrantes en que se ubicó la plataforma durante el entrenamiento.

\section{Figura 3}

Permanencia en cuadrantes reforzados

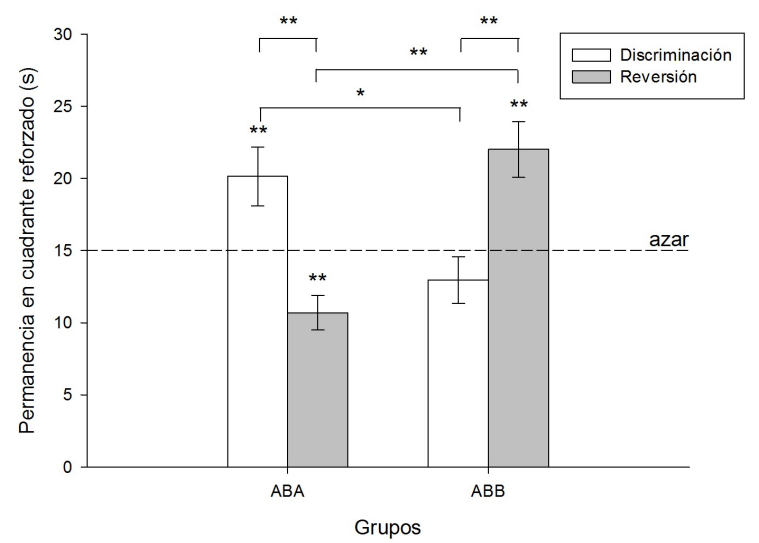

Tiempo promedio de permanencia en el cuadrante reforzado durante la Fase de Discriminación (barra blanca) y la Fase de Reversión (barra gris). La barra de error indica el error estándar de la media y la línea punteada el nivel esperado por azar. ${ }^{*}: \mathrm{p}=0.01{ }^{* *}: \mathrm{p}<0.01$. Fuente: elaboración propia. 


\section{Discusión}

El objetivo de este experimento fue evaluar la ocurrencia de un efecto de renovación de la memoria espacial en humanos entrenados en dos fases sucesivas en un LVA. Durante una Fase de Discriminación, los participantes aprendieron la ubicación de la plataforma oculta. Esto indica la adquisición de una primera memoria espacial (Hardt et al., 2009; Jacobs, Laurence \& Thomas, 1997). Durante una fase posterior (i.e., Fase de Reversión), la plataforma oculta se colocó en un sitio novedoso y nuevamente su ubicación fue aprendida, lo que demuestra la adquisición de una segunda memoria espacial (Luna \& Martínez, 2015). Este procedimiento es análogo al de aprendizaje de reversión de la discriminación utilizado en tareas no espaciales de condicionamiento y de juicio causal, y que es utilizado para ocasionar la extinción de un primer aprendizaje adquirido (Bouton \& Brooks, 1993; García-Gutierrez \& Rosas, 2003; Nakajima, Takana, Urushihara \& Imada, 2000). Para nuestro estudio, la extinción de la memoria espacial originalmente adquirida fue demostrada durante la Fase de Prueba por el Grupo ABB, el cual mantuvo una permanencia al nivel predicho por el azar en el primer cuadrante reforzado. Por el contrario, el Grupo ABA mostró una preferencia por este mismo cuadrante, lo que confirma la adquisición de una memoria espacial para la primera ubicación de la plataforma oculta. Los datos del Grupo ABA también demuestran que al realizar la prueba en un contexto diferente al presente durante la extinción (i.e., Fase de Reversión), la memoria espacial originalmente adquirida y que fue posteriormente sujeta a extinción, es recuperada. Este resultado es consistente con un efecto de renovación de la memoria espacial en humanos, que es además semejante al obtenido en otros estudios realizados con roedores (Lattal et al., 2003; Prados et al., 2003). También es coherente con demostraciones previas del efecto de recuperación espontánea de la memoria espacial en animales humanos (Luna \& Martínez, 2015) y no humanos (Lattal et al., 2003; Leising et al., 2015; Prados et al., 2003). Esto ya que ambos efectos indican que, en forma semejante a lo que sucede con otros tipos de memoria (e.g., García-Gutierrez \& Rosas, 2003), la extinción de la memoria espacial no elimina la información acerca de la ubicación de una meta, sino solamente la inhibe con posibilidad de recuperarla posteriormente.

Mecanismos cognitivos de aprendizaje asociativo han sido implicados en la adquisición de la memoria espacial (Leising \& Blaisdell, 2009). La demostración de renovación de la memoria espacial indica que estos mecanismos también participan en su extinción, lo que establece para humanos y roedores una continuidad entre ambos efectos. Tal interpretación y su evidencia, son opuestas a la noción de que la extinción ocasiona la pérdida de la información acerca de la ubicación de una meta, la cual ha sido propuesta por teorías basadas en la función del hipocampo, las cuales además excluyen la participación de aprendizaje asociativo en la memoria espacial. Por ejemplo, la teoría del mapa cognitivo (O'Keefe \& Nadel, 1978) propone que la memoria espacial se adquiere por el establecimiento de una representación cognitiva que integra todos los componentes del entorno. Ante cambios ambientales, dicha representación se actualiza para adicionar los elementos recién incorporados o eliminar la información espacial de los que se han retirado. Esta última sin posibilidad de ser recuperada. Bajo tales supuestos y dado que la extinción de la memoria espacial implica retirar del entorno la meta, esta teoría predice la pérdida de la información acerca de su ubicación. Los datos de este experimento son opuestos a dicha predicción, y pueden ser mejor explicados a partir del modelo de la recuperación de la información (Bouton, 1993; para una revisión ver Sánchez-Carrasco \& Nieto, 2009), desarrollado en el marco del aprendizaje asociativo. Desde este modelo, la adquisición ocurre por el establecimiento de una asociación excitatoria entre la representación cognitiva de un estímulo condicionado (EC) y la de un estímulo incondicionado (EI). Una vez establecida dicha asociación, la presentación del EC activa la representación del EI, provocando la respuesta condicionada (RC). Durante la 
extinción, se establece una asociación entre las representaciones del EC y el EI de naturaleza inhibitoria, lo que suprime la RC. No obstante, la asociación excitatoria original permanece intacta. Considerando que posterior a la extinción el EC predice tanto la presencia como la ausencia del EI, es necesario contar con un elemento que permita identificar la asociación vigente (i.e., excitatoria o inhibitoria). El contexto cumple esta función, de forma que la activación de la segunda asociación, en este caso la asociación inhibitoria, está modulada por el contexto en que se presenta el EC. Si el EC se ocurre en el contexto en que se llevó a cabo la extinción, entonces la asociación activa será la inhibitoria y por tanto la RC será omitida; en cambio, si el EC ocurre en un contexto distinto, entonces la asociación activa será la excitatoria y la RC será emitida. En relación al experimento aquí presentado, es plausible suponer que al menos un elemento del entorno adoptó el papel del EC en tanto que predijo la ubicación original de la plataforma oculta (EI). Entonces, durante la Fase de Discriminación pudo establecerse una asociación excitatoria entre la representación de estos, la cual produjo la RC de aproximación al lugar de meta. Para la Fase de Reversión, esta primera asociación se mantuvo intacta, aunque una asociación de tipo inhibitoria fue establecida produciendo la supresión de la RC original. Cuando la Fase de Prueba se realizó en el mismo contexto de la extinción (i.e., Fase de Reversión), la primer $\mathrm{RC}$ adquirida se omitió (Grupo ABB); pero cuando fue realizada en un contexto diferente a éste, entonces la RC original nuevamente ocurrió (Grupo ABA). Una interpretación semejante fue utilizada por Luna y Martínez (2015) para explicar el efecto de recuperación espontánea de la memoria espacial en humanos.

Nuestros resultados tienen varias limitaciones que posteriores estudios deberán considerar a fin de fortalecer la propuesta de la participación de mecanismos de aprendizaje asociativo en la extinción de la memoria espacial. Primero, existen tres diferentes tipos de renovación, a saber la renovación $\mathrm{ABA}$, la $\mathrm{ABC}$, y la $\mathrm{AAB}$ (Thomas, Larsen \& Ayres, 2003). En este experimento sólo se demostró la primera de ellas, de tal forma que futuros estudios deberán verificar la ocurrencia de las restantes así como de sus características. Segundo, existe evidencia de que la intensidad de una $\mathrm{RC}$ recuperada es menor en comparación a la intensidad de una RC que no fue extinguida (Bouton \& King, 1983). La ausencia de un grupo no expuesto a una fase de extinción impide verificar este resultado para la memoria espacial. Este aspecto deberá también ser atendido por estudios posteriores. Finalmente, cuando se emplea un laberinto de agua como tarea espacial, la permanencia en el cuadrante reforzado es un indicador de memoria espacial con sensibilidad disminuida respecto a otros indicadores (Maei, Zaslavsky, Teixeira \& Frankland, 2009). Futuros estudios sobre renovación de la memoria espacial que empleen el laberinto de agua deberán incorporar también diversos indicadores, a fin de obtener información más certera sobre los mecanismos cognitivos que operan en su extinción.

En conclusión, los resultados obtenidos en este experimento indican la ocurrencia de un efecto de renovación de la memoria espacial en humanos, lo que sugiere la participación de mecanismos propios al aprendizaje asociativo en la extinción de este tipo de memoria.

\section{Agradecimiento}

Esta investigación fue financiada a través de la beca SIP20151614 otorgada por el Instituto Politécnico Nacional, México, al proyecto "Extinción y recuperación de la conducta espacial humana en un laberinto virtual de agua" dirigido por el primer autor durante 2015. * David Luna y Moisés ManzanaresSilva colaboraron en forma equivalente en la realización del estudio.

\section{Referencias}

Aron. A., \& Aron, E. N. (2001). Estadística para psicólogos. México: Prentice Hall.

Ayaz, H., Shewokis, P. A., Curtin, A., Izzetoglu, M., Izzetoglu, K., \& Onaral, B. (2011). 
Using maze suite and functional near infrared spectroscopy to study learning in spatial navigation. Journal of Visualized Experiments, 56, e3443. doi: 10.3791/3443

Bouton, M. E. (1993). Context, time, and memory retrieval in the interference paradigms of pavlovian learning. Psychological Bulletin, 114, 80-99. doi: http: //dx.doi.org/10.1037/0033-2909.114.1.80

Bouton, M. E., \& King, D. A. (1983). Contextual control of the extinction of conditioned fear: Tests for the associative value of the context. Journal of Experimental Psychology: Animal Behavior Processes, 9, 248-265. doi: http://dx.doi.org/10.1037/0097-7403.9 .3 .248

Bouton, M., E., \& Brooks, D., C. (1993). Time and context effects on performance in a pavlovian discrimination reversal. Journal of Experimental Psychology: Animal Behavior Processes, 19, 165-179.

Burgess, N., Jackson, A., Hartley, T., \& O'keefe, J. (2000). Predictions derived from modelling the hippocampal role in navigation. Biological Cybernetics, 83, 301-312.

Devenport, L. D. (1984). Extinction-induced spatial dispersion in the radial arm maze: arrest by ethanol. Behavioral Neuroscience, 98, 979-985. doi: http://dx.doi.org/10.1037 10735-7044.98.6.979

Foster, D. J., Morris, R. G. M., \& Dayan, P. (2000). A model of hippocampally dependent navigation, using the temporal difference learning rule. Hippocampus, 10, 1-16. doi:10.1002/ (SICI) 1098-1063(2000) 10:1<1::AID$\mathrm{HIPO} 1>3.0 . \mathrm{CO} ; 2-1$

García-Gutiérrez, A., \& Rosas, J. M. (2003). Recuperación de la relación claveconsecuencia por el cambio de contexto después de la interferencia en aprendizaje causal. Psicológica, 24, 243-270.

Hampson, E. (1995). Spatial cognition in humans: Possible modulation by androgens and estrogens. Journal of Psychiatry and Neurosciences, 20, 397-404.
Hardt, O., Hupbach, A., \& Nadel, L. (2009). Factors moderating blocking in human place learning: The role of task instructions. Learning EF Behavior, 37, 42-59. doi: 10.3758/LB.37.1.42

Hardt, O., Wang, S. H., \& Nader, K. (2009). Storage or retrieval deficit: the yin and yang of amnesia. Learning $\mathfrak{E}$ Memory, 16, 224-230. doi: 10.1101/lm.1267409

Jacobs, W. J., Laurance, H. E., \& Thomas, K. G. F. (1997). Place learning in virtual space I: Acquisition, overshadowing, and transfer. Learning and Motivation, 28, 521-541. doi: 10.1006/lmot.1997.0977

Kinloch, J. M., Foster, T. M., \& McEwan, J. S. (2009). Extinction-induced variability in human behavior. The Psychological Record, 59, 347-370. http://hdl.handle.net/10289/4 573

Lattal, K. M., Mullen, M. T., \& Abel, T. (2003). Extinction, renewal and spontaneous recovery of a spatial preference in the water maze. Behavioral Neuroscience, 117, 1017-1028. doi: http:// dx.doi.org/10.1037/0735-7044.117.5.1017

Leising, K. J, \& Blaisdell, A. P. (2009). Associative basis of landmark learning and integration in vertebrates. Comparative Cognition \&ु Behavior Reviews, 4, 80-102.

Leising, K. J., Wong, J., \& Blaisdell, A. P. (2015). Extinction and spontaneous recovery of spatial behavior in pigeons. Journal of Experimental Psychology: Animal Learning and Cognition, 41, 371. doi: http://dx.doi.or $\mathrm{g} / 10.1037 / \mathrm{xan} 0000076$

Luna, D., \& Martínez, H. (2015). Spontaneous recovery of human spatial memory in a virtual water maze. Psicológica, 36, 283-308.

Mackintosh, N.J., 1983. Conditioning and Associative Learning. Oxford University Press, New York.

Maei, H. R., Zaslavsky, K., Teixeira, C. M., \& Frankland, P. W. (2009). What is the most sensitive measure of water maze probe test performance? Frontiers in Integrative Neuroscience, 3, 1-9. doi: 10.3389/neuro.07.004.2009 
Méndez-Couz, M., Conejo, N. M., Vallejo, G., \& Arias, J. L. (2015). Brain functional network changes following Prelimbic area inactivation in a spatial memory extinction task. Behavioural Brain Research, 287, 247-255. doi: 10.1016/j.bbr.2015.03.033

Méndez-Couz, M., Conejo, N. M., Vallejo, G., \& Arias, J. L., (2014). Spatial memory extinction: A c-Fos protein mapping study. Behavioural Brain Research, 260, 101-110. doi: http://dx.doi.org/10.1016/j.bbr.2013.1 1.032

Nakajima, S., Tanaka, S., Urushihara, K., \& Imada, H. (2000). Renewal of extinguished lever-press responses upon return to the training context. Learning and Motivation, 31, 416-431. doi: 10.1006/lmot.2000.1064

O'Keefe, J., \& Nadel, L. (1978). The hippocampus as a cognitive map. Oxford: Clarendon Press.

Pavlov, I. (1927). Conditioned reflexes. Inglaterra: Oxford University Press.

Prados, J., Manteiga, D., \& Sansa, J. (2003). Recovery effects after extinction in the Morris swimming pool navigation task. Learning $\mathcal{F}$ Behavior, 31, 299-304.

Pravosudov, V. V., \& Roth II, T. C. (2013). Cognitive ecology of food hoarding: The evolution of spatial memory and the hippocampus. Annual Review of Ecology, Evolution, and Systematics, 44, 173-193. doi:10.1146/ annurev-ecolsys-110512-135904

Quirk, G. J., \& Muller, D. (2008). Neural mechanisms of extinction learning and retrieval Neuropsychopharmacology, 33, 56-72. doi: 10.1038/sj.npp.1301555

Rescorla, R. A. (2001). Experimental extinction. In R. R. Mowrer \& S. B. Klein (Eds.), Handbook of contemporary learning theories (pp. 119-154). Mahwah, N. J.: Lawrence Erlbaum Associates, Inc.

Rescorla, R. A., \& Wagner, A. (1972). A theory of pavlovian conditioning: Variations in the effectiveness of reinforcement and nonreinforcement. En A. Black \& W. Prokasy (Eds.), Classical conditioning II: current research and theory (pp. 64-99). New York: Appleton-Century-Crofts.
Rossato, J. I., Bevilaqua, L. R. M., Medina, J. H., Izquierdo, I., \& Cammarota, M., (2006). Retrieval induces hippocampal-dependent reconsolidation of spatial memory. Learning Eु Memory, 13, 431-440. doi: 10.1101/ $\operatorname{lm} .315206$

Sánchez-Carrasco, L., \& Nieto, J. (2009). Recuperación de respuestas: una revisión de la evidencia y del modelo de recuperación de información. Revista Mexicana de Análisis de la Conducta, 35, 45-59.

Thomas, B. L., Larsen, N., \& Ayres, J. J. B. (2003). Role of context similarity in $\mathrm{ABA}, \mathrm{ABC}$, and $\mathrm{AAB}$ renewal paradigms: Implication $s$ for theories of renewal and for treating human phobias. Learning and Motivation, 34, 410-436. doi: 10.1016/ S0023-9690(03)00037-7

W. M. A. (World Medical Association Declaration of Helsinki) (2008). Ethical principles for medical research involving human subjects. In World Medical Association. Recuperado de http://www.wma.net/en/30publications/ 10policies/b3/index.html

Wasserman, E. A. (1993). Comparative cognition: Beginning the second century of the study of animal intelligence. Psychological Bulletin, 113, 211-228. doi: http://dx.doi.org/10.1037/0033-2909.1 13.2.211

\section{Notas}

* Artículo de investigación. 